\title{
Fallopian Tube Metaplastic Papillary Tumor
}

National Cancer Institute

\section{Source}

National Cancer Institute. Fallopian Tube Metaplastic Papillary Tumor. NCI Thesaurus.

Code C40116.

A rare metaplastic lesion that arises from the fallopian tube. It is characterized by the presence of an intraluminal papillary proliferation composed of atypical epithelial cells with abundant eosinophilic cytoplasm. In the vast majority of cases, it is an incidental finding during microscopic examination of a fallopian tube in the postpartum period. 